\title{
Inhibition of neutrophil chemotaxis by pig seminal plasma in vitro: a potential method for modulating post-breeding inflammation in sows
}

\author{
K. J. Rozeboom ${ }^{1}$, G. Rocha-Chavez ${ }^{2}$ and M. H. T. Troedsson ${ }^{3}$ \\ ${ }^{1}$ Department of Animal Science, North Carolina State University, Raleigh, NC 27695, USA; \\ ${ }^{2}$ Departamento de Produccion Animal, Universidad de Guadalajara, Cd. Guzman, \\ Jal 49000, Mexico; and ${ }^{3}$ Clinical and Population Sciences, University of Minnesota, St Paul, \\ MN 55108, USA
}

The aim of this study was to determine the regulatory role of pig seminal plasma in post-breeding uterine inflammation. Polymorphonuclear neutrophil (PMN) chemotaxis of lipopolysaccharide (LPS)-activated blood plasma or heat-inactivated blood plasma plus LPS containing increasing concentrations of seminal plasma was assessed in chemotactic chambers. Seminal plasma was diluted serially with McCoy's medium to concentrations of $50.0,25.0,12.5,6.2$ or $3.1 \%(v / v)$ and added to normal or heat-inactivated pig blood plasma that was activated with LPS before or after incubation in a $37^{\circ} \mathrm{C}$ waterbath for $30 \mathrm{~min}$. Chemotaxis was determined using blood-derived PMNs and was expressed as a percentage of the positive control of LPS-activated blood plasma. A linear dosedependent suppression of chemotaxis by seminal plasma was observed for blood plasma activated before or after addition of seminal plasma. Compared with the positive control, concentrations of seminal plasma $<6.2 \%$ failed to suppress PMN chemotaxis $(P<0.05)$. A dose-dependent suppressive effect of seminal plasma on heat stable chemotactic components of pig blood plasma was also observed $(P<0.05)$. A marked suppression was observed at concentrations of seminal plasma $>12.5 \%$ of the sample volume $(P<0.05)$. These results indicate that seminal plasma suppresses chemotactic blood plasma components regardless of formation sequence (pre- or post-activation) or source (normal or heat-inactivated blood plasma). These results indicate that seminal plasma may be necessary in diluted boar semen used for artificial insemination to regulate post-breeding inflammation in sows.

\section{Introduction}

Post-breeding inflammation in the form of a rapid migration of polymorphonuclear neutrophilic granulocytes (PMNs) into the uterus is found consistently after insemination in sows and mares (Pursel et al., 1978; Rodriguez-Martinez et al., 1990; Kotilainen et al., 1994; Troedsson, 1995; Rozeboom et al., 1998, 1999). A uterine inflammatory response to spermatozoa appears to be a normal physiological reaction after insemination (Rozeboom et al., 1998; Troedsson, 1998; Troedsson et al., 2000). Pig spermatozoa and seminal plasma appear to have equally important, yet different, modulatory roles for initiating and suppressing this response in utero (Rozeboom et al., 1999). Although a transient semeninduced uterine inflammation is probably necessary for the establishment of pregnancy (Engelhardt et al., 1997; Rozeboom et al., 1997), uncontrolled post-mating endometritis has been considered a cause of infertility in mares and pigs (McKinnon and Voss, 1993; Troedsson et al., 1995a,b; Rozeboom et al., 2000). Stallion, bull and boar spermatozoa all induce PMN chemotaxis via complement

Email: Kevin_rozeboom@ncsu.edu activation (Clark and Klebanoff, 1976; Troedsson et al., 1998; Rozeboom et al., in press). Although stallion and bull seminal plasma appear to inhibit the activation of complement, the role of boar seminal plasma has received little attention. The aims of the present study were to investigate the role of pig seminal plasma on PMN chemotaxis in vitro.

\section{Materials and Methods}

\section{Seminal plasma}

Seminal plasma was recovered from eight reproductively mature Hampshire and Yorkshire boars (pool A), and two non-related Hampshire boars from a different farm (pool B). After centrifugation at $400 \mathrm{~g}$ for $10 \mathrm{~min}$, the seminal plasma from each pool of boars was recovered and centrifuged again at $10000 \mathrm{~g}$ for $20 \mathrm{~min}$. The supernatant of each pool was recovered and $3 \mathrm{ml}$ aliquots were prepared, frozen and stored at $-20^{\circ} \mathrm{C}$ until used.

\section{Standard pooled plasma}

Peripheral blood was withdrawn, using a vacutainer system (Becton Dickinson, Rutherford, NJ) containing 
sodium heparin as an anticoagulant from the jugular vein of five clinically healthy castrated pigs (mass approximately $105 \mathrm{~kg}$ ). The blood was centrifuged at $1000 \mathrm{~g}$ for $10 \mathrm{~min}$ and the blood plasma from all pigs was saved and mixed. Aliquots $(2 \mathrm{ml})$ of the pooled blood plasma were prepared and stored at $-20^{\circ} \mathrm{C}$ until used. Untreated plasma served as a control for spontaneous chemotactic activity of blood plasma and blood plasma inactivated by heating in a $59^{\circ} \mathrm{C}$ waterbath for $40 \mathrm{~min}$ served as a negative control for complement dependent chemotactic activity.

\section{Preparation of PMNs}

A total of $20 \mathrm{ml}$ of venous blood was withdrawn from the jugular vein of a clinically healthy castrated pig (mass approximately $105 \mathrm{~kg}$ ) in a vacutainer system containing sodium heparin as an anticoagulant. The blood was centrifuged at $1000 \mathrm{~g}$ for $10 \mathrm{~min}$ and the blood plasma was removed. The buffy coat was recovered with a sterile Pasteur pipette and mixed with an isotonic saline solution $\left(9 \mathrm{~g} \mathrm{NaCl}{ }^{-1}\right)$ layered on lymphocyte separation medium (Organon Teknika, Durham, NC), and centrifuged at $1000 \mathrm{~g}$ for $20 \mathrm{~min}$. The PMN-rich buffy coat was collected from the pellet underneath the lymphocyte separation medium and re-suspended in a $2.25 \%(\mathrm{w} / \mathrm{v})$ dextran solution (average $M_{\mathrm{r}}$ 250 000; Sigma, St Louis, MO) in saline. After 15 min of sedimentation, the supernatant was collected and the cells were washed twice with saline and counted.

\section{Experiment 1}

This experiment was designed to determine the effect of seminal plasma on PMN chemotaxis as it relates to interference on activation of chemotactic substances in blood plasma by bacterial endotoxin (lipopolysaccharide (LPS); Forsberg and Carlson, 1998). A positive control was prepared mixing $40 \mu \mathrm{g}$ Escherichia coli LPS (Sigma) dissolved in $20 \mu \mathrm{l}$ saline $\left(9 \mathrm{mg} \mathrm{NaCl}{ }^{-1}\right)$ with $400 \mu \mathrm{l}$ pooled blood plasma. An additional five samples of LPS were mixed with blood plasma in the presence of 50.0, 25.0, $12.5,6.2$ and $3.1 \%(\mathrm{v} / \mathrm{v})$ seminal plasma (final seminal plasma concentrations; diluted in McCoy's modified medium (Sigma; with sodium bicarbonate and without Lglutamine)). A negative control was prepared by mixing LPS with $400 \mu \mathrm{l}$ McCoy's 5a modified medium supplemented with 10\% heat-inactivated fetal bovine serum (Gibco, Grand Island, NY). Random migration of PMNs towards seminal plasma was assayed using $400 \mu \mathrm{l}$ undiluted seminal plasma only $(100 \%)$ and an additional sample of LPS plus heat-inactivated blood plasma (heated at $56^{\circ} \mathrm{C}$ for $35 \mathrm{~min}$ ) was used as a control for non-complement related chemotactic factors. After preparation, samples were incubated at $39^{\circ} \mathrm{C}$ for $30 \mathrm{~min}$ to activate chemotactic factors in blood plasma and the process was stopped by repeated heat inactivation at $56^{\circ} \mathrm{C}$ for $35 \mathrm{~min}$ and subsequently dilution with $1.6 \mathrm{ml}$ McCoy's medium. A 48-well chemotaxis chamber (Neuroprobe Inc, Cabin John, MD) was used for all chemotaxis assays. Aliquots $(25 \mu \mathrm{l})$ of each sample were placed in corresponding wells of the bottom plate of the chamber and a $25 \mathrm{~mm} \times 80 \mathrm{~mm} 3 \mu \mathrm{m}$ pore size polycarbonate filter (Osmonics, Livermore, CA) was placed on top of the plate. After assembly of the chamber, $2.5 \times$ $10^{6}$ PMNs suspended in $50 \mu \mathrm{l} \mathrm{McCoy}$ 's medium were added to each of the wells in the upper plate. The chamber was incubated at $38^{\circ} \mathrm{C}$ with $5 \% \mathrm{CO}_{2}$ for 80 min to allow PMNs to migrate towards the chemoattractants. After incubation was complete, the chamber was inverted onto a paper towel to allow draining of the remaining cell suspension. The filter was recovered and placed upside down on a microscope slide. The treatment areas located on the filter were labelled with a permanent marker to retain identity during the staining and counting procedures. The filter was secured with clips, fixed with alcohol for $5 \mathrm{~min}$ and stained with Harris's haematoxylin and Wright stains (Sigma, St Louis, MO). The number of PMNs from eight consecutive microscopic fields was counted in different areas of the filter and the mean number of neutrophils per treatment was calculated. The chemotactic response was expressed as a percentage of the positive control (LPSactivated blood plasma). Five trials were conducted and each sample was assayed in triplicate. The means and standard error of the means were computed and registered.

\section{Experiment 2}

This experiment was designed to assess the effect of seminal plasma on PMN chemotaxis in the presence of complement-activated blood plasma. Six samples were prepared by mixing $40 \mu \mathrm{g}$ E. coli LPS (Sigma) dissolved in $20 \mu \mathrm{l}$ saline $\left(9 \mathrm{mg} \mathrm{NaCl} \mathrm{ml}^{-1}\right)$, with $400 \mu \mathrm{l}$ pooled blood plasma. All samples were incubated at $37^{\circ} \mathrm{C}$ for $30 \mathrm{~min}$, heat inactivated at $56^{\circ} \mathrm{C}$ for $35 \mathrm{~min}$ and diluted with seminal plasma to concentrations of 50.0, 25.0, $12.5,6.2,3.1$ or $0 \%$ (positive control) $(\mathrm{v} / \mathrm{v})$ seminal plasma. A negative control was prepared mixing LPS with $400 \mu \mathrm{l}$ McCoy's 5 a modified medium supplemented with $10 \%$ heat-inactivated fetal bovine serum. Random migration of PMNs towards seminal plasma was assayed using $400 \mu \mathrm{l}$ undiluted seminal plasma only $(100 \%)$ and an additional sample of LPS + heatinactivated blood plasma $\left(56^{\circ} \mathrm{C}\right.$ for $\left.30 \mathrm{~min}\right)$ was used as a control for non-complement related chemotactic factors. All samples were diluted with $1.6 \mathrm{ml}$ of McCoy's medium before being placed in the chemotactic chamber as described in Expt 1.

\section{Experiment 3}

This experiment was designed to assess the ability of seminal plasma to regulate migration of heat stable chemotactic components in pig blood plasma. Seminal plasma, blood plasma and neutrophil isolation were prepared as described earlier. However, for this experiment the blood was centrifuged for $10 \mathrm{~min}$ at $1000 \mathrm{~g}$ and the blood plasma from all pigs was removed, mixed and heat inactivated at $59^{\circ} \mathrm{C}$ for $35 \mathrm{~min}$. Aliquots $(2 \mathrm{ml})$ of the pooled blood plasma were prepared and stored at $-20^{\circ} \mathrm{C}$ until used. 
Six test tubes were prepared by mixing $40 \mu \mathrm{g} E$. coli LPS dissolved in $20 \mu \mathrm{l}$ saline $\left(9 \mathrm{mg} \mathrm{NaCl} \mathrm{ml}^{-1}\right.$ ) with $400 \mu \mathrm{l}$ heatinactivated blood plasma. Samples were incubated at $39^{\circ} \mathrm{C}$ for 30 min to activate chemotactic factors in blood plasma and the process was stopped by repeated heat inactivation at $56^{\circ} \mathrm{C}$ for $35 \mathrm{~min}$. Samples were assigned to the following treatments: addition of 50.0, 25.0, 12.5, 6.2, 3.1 and $0.0 \%$ (positive control) seminal plasma (final concentrations of seminal plasma). An additional sample was prepared as a negative control by mixing LPS with $400 \mu \mathrm{l}$ of McCoy's 5a modified medium supplemented with $10 \%$ heat-inactivated fetal bovine serum. Random migration of PMNs towards seminal plasma was assayed using $400 \mu \mathrm{l}$ undiluted seminal plasma only $(100 \%)$. All samples were diluted with $1.6 \mathrm{ml}$ McCoy's medium before being transferred to a 48well chemotaxis chamber for the chemotaxis assays. The assay was conducted as described earlier.

\section{Statistical analysis}

For each assay, the PMNs of eight consecutive microscopic fields were counted in two different areas of the filter and the mean number of neutrophils per filter was calculated. Triplicate assays were performed for each sample, and the means and standard errors of the mean were computed. The chemotactic response of each seminal component was expressed as a percentage of the positive controls (endotoxin-activated plasma).

Five replicate experiments were performed for the chemotactic assays with the exception of Expt 1, in which two additional replicates using seminal plasma from pool B were analysed separately. The mean and standard variations were expressed as a percentage of the positive control and compared by ANOVA in the linear procedures of the computerized program Statistix (1997). Bonferroni's comparison of group means was used to determine variance between groups. $P<0.05$ was considered significant.

Pearson's correlation coefficient was used to determine the relationship between concentrations of seminal plasma and its effects on chemotaxis. Logarithmic and square root transformations were used to obtain the best linear fit in the chemotaxis assays and the simple linear regression analysis was conducted to obtain the regression equation and to determine the model adequacy. The level of significance was $P<0.05$.

\section{Results}

\section{Experiment 1}

In all four experiments, the chemotactic response to seminal plasma only was similar to the negative control of McCoy's medium supplemented with $10 \%$ fetal calf serum (Figs 1-4). Heat inactivation did not significantly suppress PMN chemotaxis in Expt 1 (Fig. 3). Seminal plasma from either pool of seminal plasma caused a dose dependent suppression of chemotaxis when it was added to the samples before LPS activation of blood plasma (Figs 1

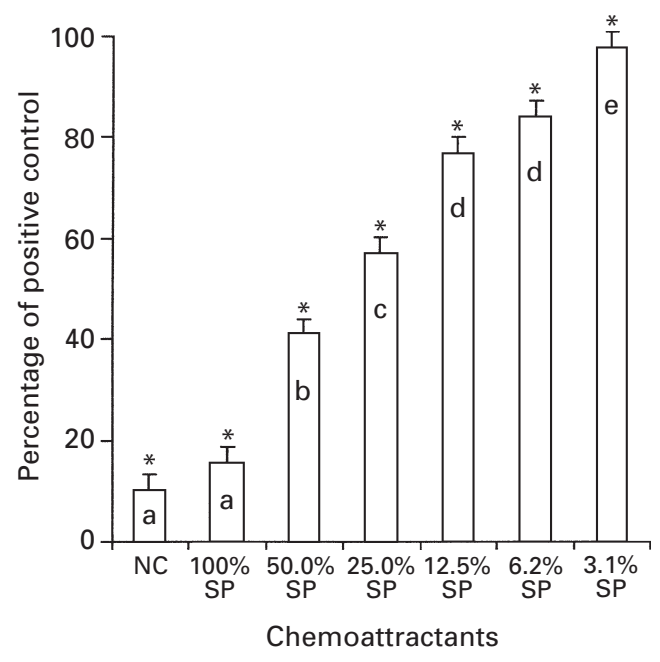

Fig. 1. Chemotaxis properties of lipopolysaccharide (LPS) plus blood plasma diluted serially with seminal plasma (SP) from boar pool A to concentrations of 50.0. 25.0, 12.5, 6.2 and $3.1 \%(\mathrm{v} / \mathrm{v})$ before incubation at $37^{\circ} \mathrm{C}$ for $30 \mathrm{~min}$ (pre-activation; Expt 1). Seminal plasma caused a dose-dependent suppression of chemotaxis when it was added to the samples before LPS activation of blood plasma. Compared with the positive control containing LPS + blood plasma only, a marked suppression of chemotaxis occurred when seminal plasma was added at $6.2 \%$ of the sample volume, whereas maximum suppression of chemotaxis occurred when seminal plasma was added at $50.0 \%$ of sample volume. NC: negative control of McCoy's medium + fetal calf serum. 100\% SP: undiluted seminal plasma used to assay random migration of PMNs towards seminal plasma. Data are expressed as percentages of the positive control. abcdeValues with different letters are significantly different $(P<0.01)$. $*$ Value is significantly different from positive control $(P<0.05)$.

and 2; $P<0.05)$, and a strong negative relationship $(r=-0.96, P<0.01)$ was found between the concentration of seminal plasma in the treatment and the percentage of chemotaxis observed. The equation of the regression line was $y=(9.55-0.058 x)^{2}$ and the coefficient of determination was $r^{2}=0.97(P<0.01)$.

\section{Experiment 2}

Increasing concentrations of seminal plasma caused a reduction in chemotactic activity of LPS-activated blood plasma when seminal plasma was added after activation (Fig. 4; $P<0.05$ ). The correlation was also significant $(r=-0.92, P<0.01)$ and the equation of the regression line was $y=(8.45-0.052 x)^{2}$, with a coefficient of determination of $r^{2}=0.90(P<0.01)$.

\section{Experiment 3}

In this experiment, a dose-dependent suppression of chemotaxis was also observed for LPS plus heat-inactivated blood plasma in the presence of increasing concentrations of seminal plasma $(P<0.05$; Fig. 5). Samples containing $\geqslant 12.5 \%(\mathrm{v} / \mathrm{v})$ seminal plasma showed a significantly 


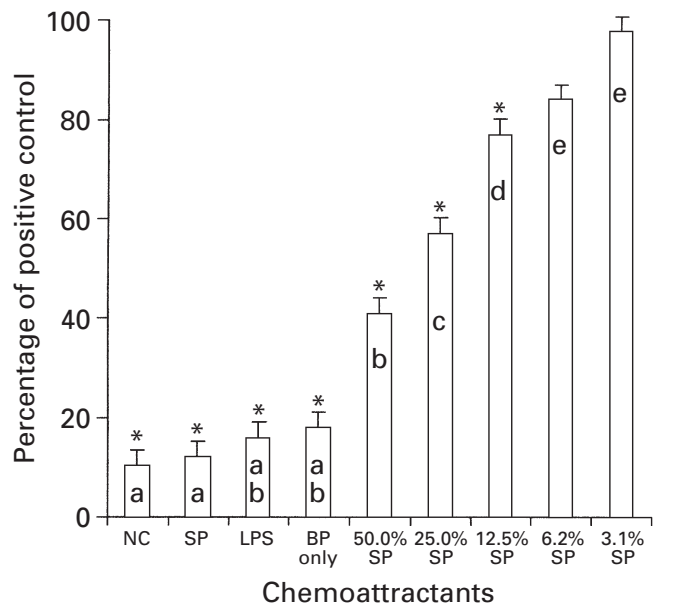

Fig. 2. Chemotaxis properties of lipopolysaccharide (LPS) plus blood plasma diluted serially with seminal plasma (SP) from boar pool B to concentrations of 50.0. 25.0, 12.5, 6.2 and $3.1 \%(\mathrm{v} / \mathrm{v})$ before incubation at $37^{\circ} \mathrm{C}$ for $30 \mathrm{~min}$ (pre-activation). Addition of seminal plasma caused a dose-dependent suppression of chemotaxis when it was added to the samples before LPS activation of blood plasma. Compared with the positive control of LPS plus blood plasma, a marked suppression occurred when seminal plasma was added at $12.5 \%$ of sample volume, whereas maximum suppression of chemotaxis occurred when seminal plasma was added at $50.0 \%$ of sample volume. Migration of PMNs toward undiluted seminal plasma (SP), LPS and blood plasma (BP) was similar to that of the negative control (NC) of McCoy's medium + fetal calf serum. Data is expressed as a percentage of the positive control. abcdeValues with different letters are significantly different $(P<0.05)$. *Value is significantly different from positive control $(P<0.05)$.

greater suppression of chemotaxis compared with the positive control $(P<0.01)$. The sample containing heatinactivated blood plasma caused only mild chemotaxis compared with the LPS + heat-inactivated blood plasma sample (positive control). The equation $y=78.71+0.81 x$ represents the regression of seminal plasma concentration on the sample $(y)$ and the amount of chemotaxis observed $(x)$. A strong negative relationship was found between the parameters $(r=-0.92, P<0.001)$.

\section{Discussion}

Extensive dilution of semen is used routinely for artificial insemination (Crabo and Dial, 1992). Previous data from our laboratory indicate that the physiological clearing processes of semen and bacterial contamination from the female reproductive tract are altered during these practices. Rozeboom et al. (1998) characterized post-breeding uterine neutrophil migration in gilts and showed that this inflammatory response (as measured by uterine neutrophil migration) reaches its magnitude between 6 and $12 \mathrm{~h}$ after artificial insemination and persists for as long as $24 \mathrm{~h}$ after

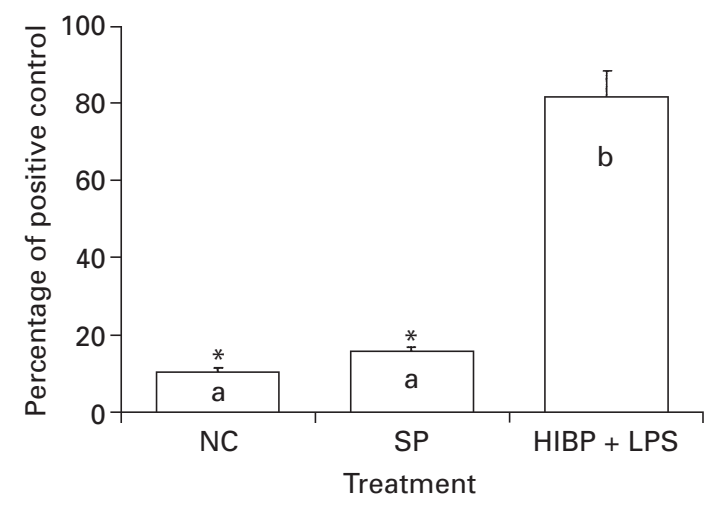

Fig. 3. Polymorphonuclear neutrophil (PMN) chemotactic properties of seminal plasma and heat-inactivated blood plasma plus lipopolysaccharide (HIBP + LPS). Chemotaxis is expressed as a percentage of positive control (LPS activated blood plasma). Migration of PMNs toward undiluted seminal plasma (SP) was similar to that of the negative control (NC) of McCoy's medium plus fetal calf serum and markedly lower than that of HIBP plus LPS and the positive control of LPS-activated blood plasma. abValues with different letters are significantly different $(P<0.01)$. Value is significantly different from positive control $(P<0.05)$.

artificial insemination. More recent studies supported this, but also suggested that the components of semen, spermatozoa and seminal plasma have different roles in the initiation, suppression and clearance of uterine neutrophil migration (Rozeboom et al., 1999). Although PMN influxes into the uterus after insemination can persist for as long as $36 \mathrm{~h}$, both the magnitude and clearance time is highly dependent on the presence or absence or seminal plasma in the dose used for artificial insemination (Rozeboom et al., 1999).

The modulatory role of seminal plasma is perhaps most evident in current semen dilution practices when doses of semen that are free of seminal plasma are used for artificial insemination. Rozeboom et al. (2000) showed that if artificial insemination doses free from seminal plasma are deposited into a neutrophil-enriched uterine environment (sperm- or endotoxin-initiated), the likelihood of a successful pregnancy is lowered significantly. These results indicate that, in addition to controlling uterine PMN migration, seminal plasma may have a protective role in sperm transport. The mechanism of the protective role of seminal plasma on sperm survival in an inflamed uterine environment in vivo is not understood. The results of the present study indicate that pig seminal plasma can interfere with complement activation and suppress the activity of blood components causing chemotaxis. However, it is not completely clear from these data whether seminal plasma inhibits the cleavage of these components (probably C3a and $\mathrm{C} 5 \mathrm{a}$ ) or simply suppresses their activity after activation. These data indicate that seminal plasma suppresses neutrophil chemotaxis in a dose-dependent fashion in vitro. 


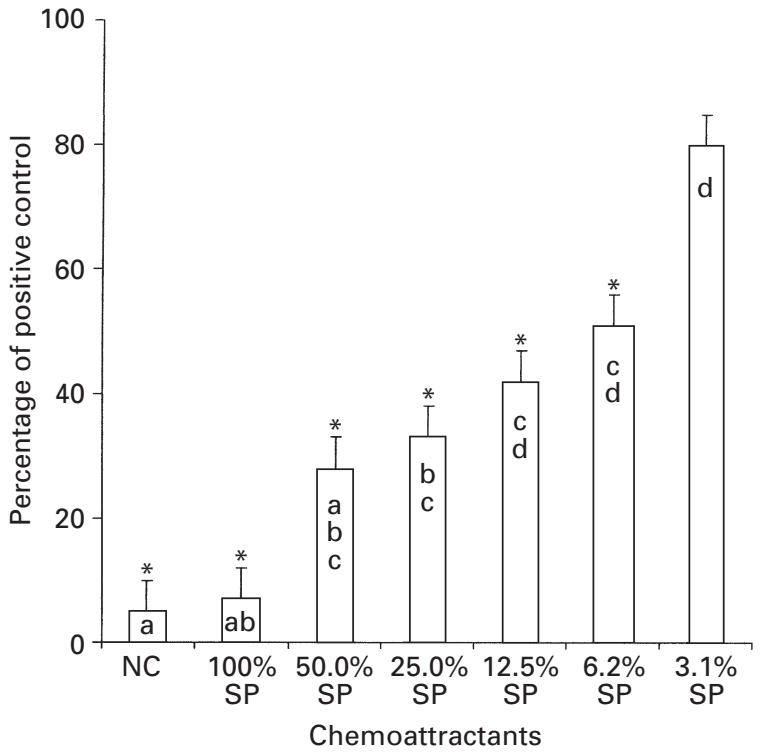

Fig. 4. Chemotaxis properties of lipopolysaccharide (LPS) plus blood plasma diluted serially with seminal plasma (SP) to concentrations of 50.0. 25.0, 12.5, 6.2 and $3.1 \%(\mathrm{v} / \mathrm{v})$ after incubation at $37^{\circ} \mathrm{C}$ for $30 \mathrm{~min}$ (post-activation; Expt 2). Addition of seminal plasma caused a dose-dependent suppression of chemotaxis when it was added to the samples before LPS activation of blood plasma. Compared with the positive control of LPS plus blood plasma, a marked suppression of chemotaxis occurred when seminal plasma was added at $6.2 \%$ of sample volume, whereas maximum suppression of chemotaxis occurred at $50.0 \%$ seminal plasma inclusion rates. NC: negative control of McCoy's medium + fetal calf serum. Data are expressed as a percentage of the positive control. 100\% SP: undiluted seminal plasma used to assay random migration of PMNs towards seminal plasma abcdValues with different letters are significantly different $(P<0.01) .{ }^{*}$ Value is significantly different from positive control $(P<0.05)$

A strong negative relationship was found between seminal plasma concentrations in the treatment and the percentage of chemotaxis observed when seminal plasma was added to blood plasma before complement activation. When seminal plasma was added after complement activation, a slightly less significant, but similar, relationship was found. Increasing concentrations of seminal plasma in heatinactivated blood plasma also caused reduction of PMN chemotaxis in a dose-dependent way. Normally, LPS activates complement via the alternative pathway in the absence of antibodies (Forsberg and Carlson, 1998), which has been a consistent in vitro response observed in our laboratory using horse serum (Troedsson et al., 1999). However, in the present study it was observed that a mixture of LPS and heat-inactivated pig blood plasma also caused a strong chemotactic response, whereas heatinactivated blood plasma provoked only mild chemotaxis. These results discard complement components as the sole chemotactic factor in pigs and indicate that LPS can activate other factors in blood plasma that stimulate a strong

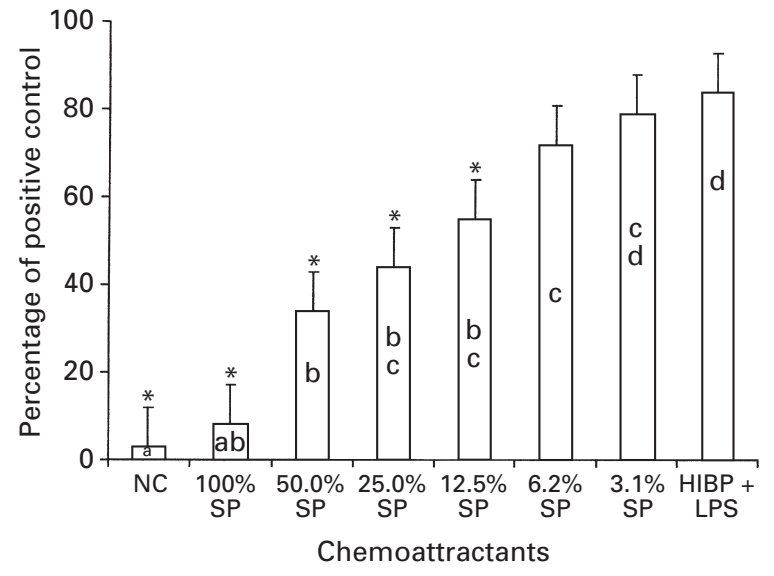

Fig. 5. Chemotaxis properties of lipopolysaccharide (LPS) plus heat inactivated blood plasma (HIBP; $59^{\circ} \mathrm{C}$ for $30 \mathrm{~min}$ ) diluted serially with seminal plasma to concentrations of 50.0. 25.0, 12.5, 6.2 and $3.1 \%(\mathrm{v} / \mathrm{v})$ after incubation at $37^{\circ} \mathrm{C}$ for $30 \mathrm{~min}$ and heat treatment of $59^{\circ} \mathrm{C}$ for 35 min (Expt 3). Seminal plasma (SP) caused a dose-dependent suppression of chemotaxis when it was added to the samples after LPS stimulation of heat stable chemotactic blood plasma components. Compared with the positive control of LPS plus HIBP, a marked suppression of chemotaxis occurred when seminal plasma was added at $12.5 \%$ of sample volume, whereas maximum suppression of chemotaxis occurred when seminal plasma was added at $50.0 \%$ of sample volume. NC: negative control of McCoy's medium + fetal calf serum. 100\% SP: undiluted seminal plasma used to assay random migration of polymorphonuclear neutrophils towards seminal plasma. Data are expressed as a percentage of the positive control. abcdValues with different letters are significantly different $(P<0.01)$. *Value is significantly different from positive control $(P<0.05)$.

chemotactic response, as LPS alone does not elicit a PMN chemotactic response (K. J. Rozeboom, unpublished). A potential explanation for this event is that isolation of neutrophils was never accomplished at $100 \%$ purity and that other blood cells such as monocytes $(12 \%)$ and some lymphocytes $(15 \%)$ were present in the solution. Bloodderived PMNs rather than uterine derived PMNs were used for all chemotaxis experiments. Endometritis would have to be induced experimentally to obtain uterine derived PMNs, as very few PMNs are normally present in a healthy nonchallenged uterus. PMNs obtained from an in vivo inflammation represent a heterogeneous sample of freshly recruited already phagocytosing and degenerating cells. In contrast, blood-derived PMNs can be stimulated in vitro and, therefore, are more suitable for a standardized experimental protocol in a function assay. LPS may stimulate monocytes to produce cytokines that may interact with blood plasma proteins or act directly as neutrophil activators or chemotactic agents (Abbas et al., 1997).

These studies are the first to demonstrate the ability of seminal plasma to suppress neutrophil chemotaxis in pigs in vitro. Previous investigations have reported both inhibitory and stimulatory chemotactic responses from 
human, guinea-pig, bovine and equine seminal plasma in the presence of blood plasma (Maroni et al., 1972; Clark and Klebanoff, 1976; Troedsson et al., 1998). Most of these investigations support our findings, with the exception of Clark and Klebanoff (1976), who found that bovine seminal plasma induces chemotactic activity when incubated with serum. It is difficult to speculate on variations in immunosuppressive activity between species, males within the same species or ejaculates from the same male, as the aims of the present study were to elucidate the general chemotactic properties of pig seminal plasma. Further research is needed to identify potential individual variation with respect to individual boars or ejaculates from the same boar, as it may explain some of the variability in reproductive performance when homogeneous matings are used.

The specific factors or components of seminal plasma that are responsible for reduction of chemotaxis in sows are unclear at this point; however, a protein or group of proteins may be involved in these beneficial effects. Troedsson et al. (1999) suggested that a protein fraction in stallion seminal plasma between $50 \mathrm{kDa}$ and $100 \mathrm{kDa}$ molecular mass is probably responsible for suppressing PMN chemotaxis, but more studies are needed before the potential to modulate this response artificially becomes apparent.

In conclusion, the results of the present study demonstrate an important role of seminal plasma in modulating two important events of post-breeding inflammation in sows. The results indicate that seminal plasma is needed in the uterine lumen after mating to ensure a suitable environment for spermatozoa and pre-implantation when multiple inseminations are used for artificial insemination of pigs.

\section{References}

Abbas AK, Lichtman AH and Pober JS (1997) Cytokines. In Cellular and Molecular Immunology pp 250-276. WB Saunders, Philadelphia

Clark RA and Klebanoff SJ (1976) Generation of a neutrophil chemotactic agent by spermatozoa: role of complement and regulation by seminal plasma factors Journal of Immunology 117 1378-1389

Crabo BG and Dial GD (1992) Artificial insemination in swine Veterinary Clinics of North America Food Animal Practitioner 8 533-544

Engelhardt H, Croy BA and King G (1997) Role of uterine immune cells in early porcine pregnancy Journal of Reproduction and Fertility Supplement 52 115-131

Forsberg LS and Carlson RW (1998) The structures of LPSs from Rhizobium etli strains CE358 and CE 359 Journal of Biological Chemistry 273 $2747-2757$
Kotilainen T, Huhtinen M and Katila T (1994) Sperm induced leukocytosis in the equine uterus Theriogenology 41 629-636

McKinnon AP and Voss JL (1993) Breeding the problem mare. In Equine Reproduction pp 368-378 Eds AO McKinnon and JL Voss. Lea and Febiger, Philadelphia

Maroni ES, Symon DNK and Wilkinson PC (1972) Chemotaxis of neutrophil leucocytes toward spermatozoa and seminal fluid Journal of Reproduction and Fertility Supplement 28 359-368

Pursel VG, Schulman LL and Johnson LA (1978). Distribution and morphology of fresh and frozen-thawed sperm in the reproductive tract of gilts after Al Biology of Reproduction 19 69-76

Rodriguez-Martinez H, Nicander L, Viring S, Einarsson S and Larsson $\mathrm{K}$ (1990) Ultrastructure of the uterotubal junction in preovulatory pigs Anatomia Histologia Embryologia 19 16-36

Rozeboom KJ, Troedsson MHT, Shurson GC, Hawton JD and Crabo BG (1997) Late estrus or metestrus insemination subsequent to estrual inseminations decreased farrowing rate and litter size in swine Journal of Animal Science 75 2323-2327

Rozeboom KJ, Troedsson MHT and Crabo BG (1998) Characterization of the post-breeding uterine leukocyte migration in the gilt Journal of Reproduction and Fertility 114 195-199

Rozeboom KJ, Troedsson MHT, Molitor TW and Crabo BG (1999) The effect of spermatozoa and seminal plasma on leukocyte migration into the uterus of gilts Journal of Animal Science 7 2323-2327

Rozeboom KJ, Troedsson MHT, Hodson HH, Shurson GC and Crabo BG (2000) The importance of seminal plasma on the fertility of subsequent artificial inseminations in swine Journal of Animal Science 78 443-448

Rozeboom KJ, Troedsson MHT, Rocha G and Crabo BG (2001) The chemotactic properties of porcine seminal components toward neutrophils Journal of Animal Science $\mathbf{7 9}$

Troedsson MHT (1995) Uterine response to semen deposition in the mare Proceedings of the Society of Theriogenology 130-135

Troedsson MHT, Steiger BN, Ibrahim NM, King VL, Foster DN and Crabo BG (1995a) Mechanism of sperm-induced endometritis in the mare Biology of Reproduction 52 (Supplement 1) 133 (Abstract)

Troedsson MHT, Crabo BG, Ibrahim NM, Scott M and Ing M (1995b) Mating-induced endometritis (MIE): mechanisms, clinical importance and consequences Proceedings of the American Association of Equine Practitioners 41 11-12

Troedsson MHT, Liu IKM and Crabo BG (1998) Sperm transport and survival in the mare Theriogenology 49 905-915

Troedsson MHT, Franklin RD and Crabo BG (1999) Suppression of PMN chemotaxis by different molecular weight fractions of seminal plasma Pferdeheilkunde 15 568-573

Troedsson MHT, Lee C-S, Franklin RD and Crabo BG (2000) The role of seminal plasma in post-breeding uterine inflammation Journal of Reproduction and Fertility Supplement 56 341-349

Received 7 August 2000.

First decision 25 August 2000.

Final revision received 13 November 2000.

Accepted 1 December 2000. 